\title{
Atividade da catalase e da lactato desidrogenase em tilápias submetidas a estresse de confinamento: efeito da cor do ambiente
}

\author{
Catalase and lactate dehydrogenase activity in tilapia subjected to contention stress: effect of the \\ background color
}

\author{
Elyara Maria Pereira-da-Silva ${ }^{{ }^{*}}$ Mariza Pires de MeloI ${ }^{\mathrm{II}}$ Ricardo Henrique Franco de Oliveira ${ }^{\mathrm{I}}$ \\ Silvana Marina Piccoli Pugine ${ }^{I I}$
}

\section{RESUMO}

Avaliaram-se os efeitos da cor do ambiente sobre o crescimento e a atividade da enzima antioxidante catalase (CAT) e da lactato desidrogenase ( $L D H)$ em tilápias do Nilo $(n=24 ; 36,2 \pm 3,6 g)$. Oito exemplares foram mortos para determinação da atividade basal das enzimas e os demais permaneceram isolados durante 14 dias sob espectro de luz branca ou azul ( $n=8$ peixes/tratamento). A seguir os peixes foram submetidos a um estresse diário de confinamento de 90 minutos (15ㅇ ao 28ㅇ dia) e pesados semanalmente para cálculo da taxa de crescimento específico (TCE). A TCE negativa confirmou que o confinamento provocou estresse nos peixes, independentemente da cor do ambiente. O aumento da atividade da $L D H$ no músculo vermelho dos peixes mantidos sob luz branca ou azul indicou mudança do metabolismo aeróbio para anaeróbio. $O$ estresse reduziu a atividade da CAT no músculo branco dos peixes mantidos sob a luz branca ou azul. Na musculatura vermelha, esta redução ocorreu apenas nos animais mantidos sob a luz branca. O confinamento aumenta os processos metabólicos anaeróbios e é adequado para estudos sobre os efeitos do estresse. O espectro de luz azul não evita a redução do crescimento e a demanda energética anaeróbia em situações de estresse, mas preserva a atividade da CAT, contribuindo para o bem-estar da tilápia.

Palavras-chave: confinamento, crescimento, estresse oxidativo, metabolismo, Oreochromis niloticus.

\section{ABSTRACT}

We assess the effects of the background color on the growth and antioxidant enzyme catalase (CAT) and lactate dehydrogenase $(L D H)$ in Nile tilapia $(n=24 ; 36.2 \pm 3.6 g)$. Eight fish were killed for assessment of basal activity of the enzymes and the others remained isolated for 14 days under white or blue light spectrum ( $n=8$ fish/treatment). Then each animal were subjected to a daily stress of confinement of 90 minutes $\left(15^{\text {th }}\right.$ to $28^{\text {th }}$ day) and weighed to calculate the specific growth rate (SGR). The negative SGR confirmed that the confinement stressed in fish, regardless of the background color. The increased activity of $L D H$ in red muscle of fish kept under white or blue light indicated replacement of aerobic metabolism to anaerobic. Stress reduced the CAT activity in white muscle of fish kept under white or light blue. In red muscle this reduction occurred only in animals kept under white light. Restraint causes increase in anaerobic metabolic processes and is suitable for studies on the effects of stress in tilapia. Confinement increases the anaerobic metabolic processes and is suitable for studies on the effects of stress. The blue spectrum of light does not prevent the reduction of growth and the anaerobic energetic demand in stressful situations, but preserves the CAT activity, contributing to the tilápia welfare.

Key words: confinement, growth, metabolism, Oreochromis niloticus, oxidative stress.

\section{INTRODUÇÃO}

As características espectrais da cor do ambiente afetam o status fisiológico, o comportamento e, portanto, o crescimento dos peixes cultivados, mas seus efeitos têm sido pouco investigados (KARAKATSOULI et al., 2008). PAPOUTSOGLOU et al. (2000) demonstraram que carpas mantidas em substrato de cor preta apresentaram aumento pronunciado dos níveis de cortisol plasmático, de lipídios totais no sangue, do $\mathrm{pH}$ e da taxa de conversão

ILaboratório de Comportamento de Peixes, Departamento de Ciências Básicas, Faculdade de Zootecnia e Engenharia de Alimentos (FZEA), Universidade de São Paulo (USP), 13635-900, Pirassununga, SP, Brasil. E-mail: elyara@usp.br. *Autor para correspondência. "Laboratório de Química Biológica, Departamento de Ciências Básicas, FZEA, USP, Pirassununga, SP, Brasil. 
alimentar, além de redução da taxa de crescimento, da gordura hepática e $\mathrm{pCO}_{2}$ sanguíneo. Em Oreochromis niloticus, a luz azul no ambiente evita o clássico aumento dos níveis de cortisol plasmático induzido pelo estresse (VOLPATO \& BARRETO, 2001).

Outros trabalhos reforçam os efeitos modulatórios da cor do ambiente sobre as respostas fisiológicas de várias espécies de peixes. Por exemplo, substratos de cor branca ou preta modulam comportamentos de dominância e submissão, por meio das alterações da pigmentação corpórea mediada pelo hormônio estimulador dos melanócitos (HÖGLUND et al., 2002) e afetam o crescimento corpóreo e consumo de alimento (SUNUMA et al., 2009), a composição dos ácidos graxos hepáticos (KARAKATSOULI et al., 2007) e os níveis cerebrais do hormônio liberador da gonadotropina (GnRH) e do hormônio concentrador da melanina (MCH), que interferem na taxa de ingestão alimentar (AMIYA et al., 2008). Estas mesmas cores de substrato podem, também, afetar a liberação de cortisol decorrente do aumento da densidade de estocagem (DOOLAN et al., 2009).

Considerando-se outras cores de ambiente, a luz azul é a mais investigada e, embora seus efeitos benéficos sejam descritos para os peixes, muitas contradições ainda persistem. Esta cor aumenta a agressividade ou estresse em peixes territoriais como O. niloticus (FANTA, 1995; MERIGHE et al., 2004), reduz o crescimento em Perca flavescens (HEAD \& MALISON, 2000) e evita o aumento dos níveis plasmáticos de cortisol em resposta ao estresse em $\boldsymbol{O}$. niloticus VOLPATO \& BARRETO, 2001), Rhamdia quelen (BARCELLOS et al., 2006), Pagrus pagrus (VAN DER SALM et al., 2004) e Onchorhynchus mykiss (KARAKATSOULI et al. 2008). O espectro de luz azul inibe a mobilização de lipídeos hepáticos induzida pelo estresse em O. mykiss (KARAKATSOULI et al., 2008), aumenta a eficiência alimentar em larvas de Melanogrammus aeglefinus (DOWNING \& LITVAK, 2001) e favorece o crescimento em Perccottus glenii e Poecilia reticulata, efeito não observado em Carassius carassius (RUCHIN, 2004). Por outro lado, reduz o crescimento em $\boldsymbol{P}$. flavescens, comparativamente à luz vermelha (HEAD \& MALISON, 2000), e estimula a reprodução em $\boldsymbol{O}$. niloticus (VOLPATO et al., 2004).

As espécies reativas de oxigênio como $\mathrm{O}_{2}^{-}$, $\mathrm{H}_{2} \mathrm{O}_{2}$ e $\mathrm{OH}$ são subprodutos continuamente gerados em decorrência dos processos de produção mitocondrial de ATP nos organismos (STOREY, 1996; HALLIWELL \& GUTTERIDGE, 2007). De acordo com ADAMS et al. (2000), provocam danos às proteínas, membranas e DNA, tornando necessária a atuação de sistemas enzimáticos e não enzimáticos de proteção, denominados defesas antioxidantes, que atuam na prevenção da formação das espécies reativas de oxigênio, na sua inativação por meio da combinação com enzimas antioxidantes e no reparo de danos celulares já ocorridos. O desequilíbrio entre a quantidade de espécies reativas de oxigênio produzidas e a capacidade de detoxificação do organismo pelas enzimas antioxidantes (superoxido dismutase - SOD, glutationa peroxidase - $\mathrm{GP}_{\mathrm{x}}$, catalase CAT, glutationa S-transferase - GST e outros sequestradores de baixo peso molecular como glutationa - GSH) caracteriza o estresse oxidativo (STOREY, 1996; YONAR \& SAKIN, 2011).

Embora GAINGNON et al. (1993) tenham demonstrado em Salmo salar efeito negativo da luz amarela sobre a atividade da ATPase, enzima relacionada à degradação de ATP durante o metabolismo energético da célula, trabalhos relacionados especificamente aos efeitos do espectro luminoso ambiental sobre o metabolismo oxidativo e as defesas antioxidantes dos peixes não foram publicados. Portanto, o objetivo deste trabalho foi avaliar os efeitos do espectro de luz azul do ambiente sobre o crescimento e atividade da enzima antioxidante CAT e da enzima LDH em tilápias submetidas a um estresse crônico e intenso.

\section{MATERIAL E MÉTODOS}

Machos adultos de tilápia do Nilo, Oreochromis niloticus $(\mathrm{n}=24 ; 36,2 \pm 3,6 \mathrm{~g})$, foram mantidos durante 14 dias em isolamento visual e sob luz ambiente em unidades experimentais (20L) para aclimatação. A temperatura foi mantida em $27,5^{\circ} \mathrm{C}$, o fotoperíodo em 12 horas (6:00h às 18:00h), o oxigênio acima de 4,0 $\mathrm{mg} \mathrm{L}^{-1}$, pH 6,7 e níveis reduzidos de amônia $\left(<0,5 \mathrm{mg} \mathrm{L}^{-1}\right)$ e de nitrito $\left(<0,05 \mathrm{mg} \mathrm{L}^{-1}\right)$. A alimentação consistiu em ração comercial extrusada (32\% PB) oferecida duas vezes ao dia até saciedade aparente.

Após a aclimatação, oito peixes foram anestesiados com 2-fenoxietanol (éter monofenílico de etilenoglicol; $0,5 \mathrm{~mL} \mathrm{~L}^{-1}$ de água) e mortos por decapitação para remoção de amostras de musculatura epaxial branca e vermelha (lado esquerdo), armazenadas em nitrogênio líquido para posterior avaliação da atividade basal das enzimas catalase (CAT) e lactato desidrogenase (LDH). Os demais peixes permaneceram isolados e foram submetidos durante 14 dias consecutivos (pré-estresse: $1^{ } \stackrel{0}{ }$ ao $14^{\circ} \stackrel{\circ}{ }$ dia do experimento) a dois tratamentos, caracterizados pela permanência em ambiente com espectro de luz branca ou azul ( $n=8$ peixes tratamento).

O ambiente azul foi obtido por meio do revestimento dos aquários (inclusive acima da superfície da água) com uma camada de celofane azul que proporcionou uma iluminação de 110Lux e o branco, pela sobreposição de camadas de celofane transparente na quantidade necessária para o estabelecimento da mesma intensidade luminosa. 
No início e ao final do período pré-estresse, todos os peixes foram pesados e, a partir do $15^{\circ}$ dia do experimento, cada animal foi submetido durante 90 minutos a um estresse diário de confinamento (período da manhã), caracterizado pela manutenção do peixe num compartimento plástico translúcido (13x9x5cm), imerso na água da unidade experimental e recoberto por uma tela plástica para permitir as trocas gasosas e impedir a fuga. Durante este período de estresse (15o ao $28^{\circ}$ dia do experimento), os peixes foram pesados semanalmente (balança eletrônica de precisão) para o cálculo da taxa de crescimento específico (TCE), de acordo com JOBLING (1994): TCE =100 x [(ln Pf - $\ln \mathrm{Pi}$ )/ ÄT], em que Pi e Pf sao, respectivamente, os pesos iniciais e finais dos peixes e $\Delta \mathrm{T}$ o tempo. Ao final do experimento, obtiveram-se três valores da TCE (préestresse: $1^{\circ}-14^{\circ}$ dia; estresse: $15^{\circ}-21^{\circ}$ e $22^{\circ}-28^{\circ}$ dia).

No final do experimento, todos os peixes foram anestesiados com 2-fenoxietanol $\left(0,5 \mathrm{~mL} \mathrm{~L}^{-1}\right)$ e mortos por decapitação para uma segunda amostragem dos tecidos musculares e avaliação dos efeitos do estresse e da cor do ambiente sobre as atividades enzimáticas, conforme descrito para os peixes do grupo controle. Para avaliação da atividade da CAT e LDH, as amostras dos tecidos musculares foram homogenizadas em solução resfriada de tampão fosfato $10 \mathrm{mM}, \mathrm{pH} 7,4$, utilizando-se um homogenizador universal (1 $\mathrm{g}$ de tecido $10 \mathrm{~mL}^{-1}$ do tampão), de acordo com LINS et al. (2006). A atividade da CAT foi medida a 240nm, seguindo-se a redução da
$\mathrm{H}_{2} \mathrm{O}_{2}$ (BEERS \& SIZER, 1952). A atividade da LDH foi analisada seguindo-se as mudanças de absorbância a 340nm (BERNT \& BERGMEYER, 1974). Todos os reagentes foram obtidos da Sigma Chemical Company (St. Louis, MO, USA).

Os dados apresentaram distribuição normal (Shapiro-Wilks) e foram comparados por ANOVA, num delineamento inteiramente casualizado (pesos e atividade das enzimas para cada tratamento) ou medidas repetidas (taxa de crescimento específico nos três períodos considerados), conforme procedimento GLM do programa computacional Statistical Analysis System SAS (1999), comparando-se as médias pelo teste $\mathrm{t}$ de Student $(\mathrm{P}<0,05)$.

\section{RESULTADOS E DISCUSSÃO}

A qualidade da água permaneceu adequada à manutenção da tilápia e não diferiu entre os tratamentos $\left(\mathrm{pH}=6,7 \pm 0,4\right.$ e $6,7 \pm 0,3$; temperatura $=27,5 \pm 0,5^{\circ} \mathrm{C}$ e $27,6 \pm 0,4^{\circ} \mathrm{C}$, respectivamente, para a luz branca e azul). Os pesos iniciais dos peixes eram semelhantes $(36,2 \pm 3,6 \mathrm{~g})$ e não diferiram entre os tratamentos (luz branca: $37,1 \pm 2,3 \mathrm{~g}$ e luz azul $35,3 \pm 4,0 \mathrm{~g} ; \mathrm{t}=0,70 ; \mathrm{P}=0,50$ ), durante o período de pré-estresse ( $1^{\circ}$ ao $14^{\circ}$ dia).

As taxas de crescimento específico (TCE) durante o período pré-estresse foram positivas e similares entre os animais mantidos sob luz branca ou azul (Figura 1; $\mathrm{t}=1,49 ; \mathrm{P}=0,16$ ). Porém, o estresse imposto

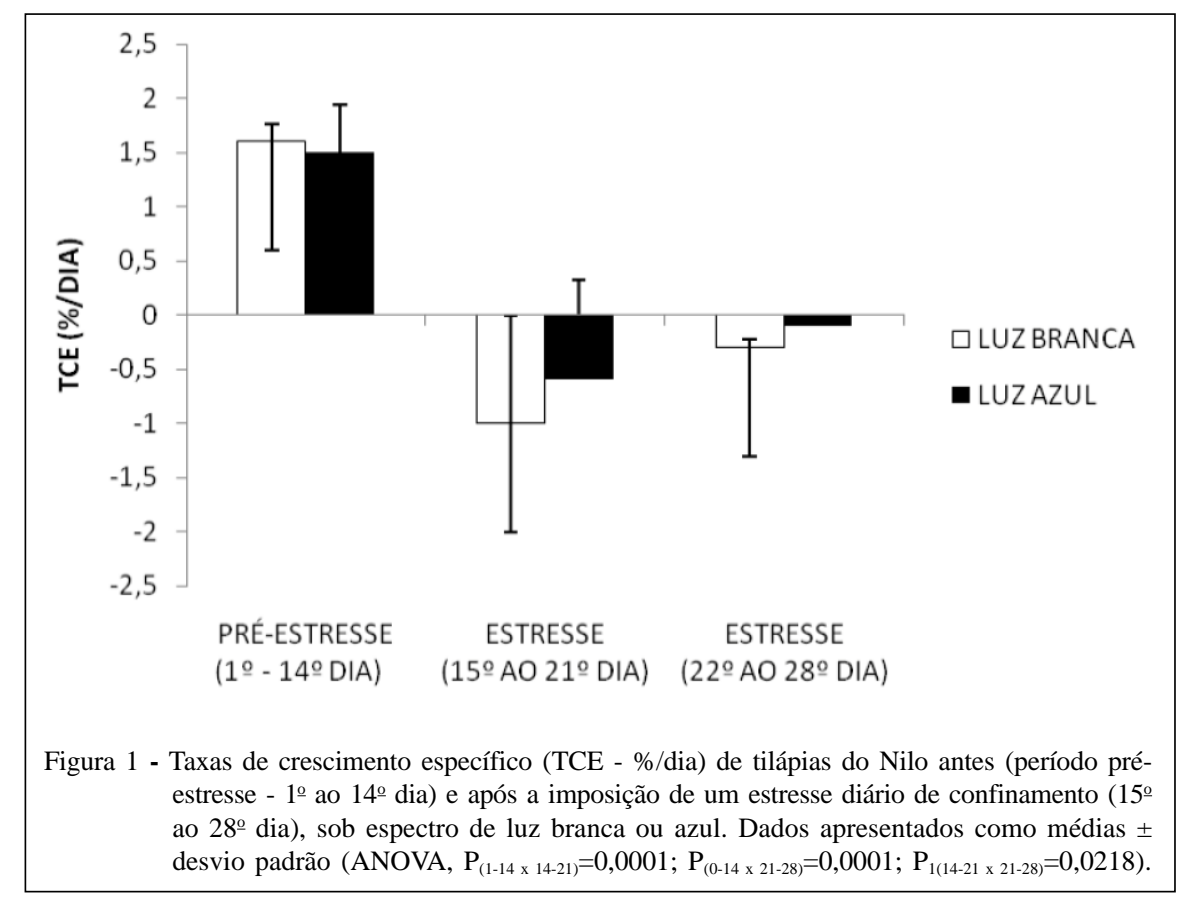

Ciência Rural, v.42, n.5, mai, 2012. 
entre o 15ㅇ e 28을 dia reduziu a TCE, independentemente do regime de espectro luminoso, comprovando a eficiência do agente estressor utilizado.

A coloração dos tanques afeta as respostas ao estresse e o crescimento de peixes como Cyprinus carpio e O. mykiss (PAPOUTSOGLOU et al., 2000 e 2005). OWEN et al. (2010) observaram estresse fisiológico mais pronunciado em juvenis de Tinca tinca mantidos durante uma semana sob luz branca, comparativamente aos que permaneceram sob luz azul. VOLPATO \& BARRETO (2001) observaram que a cor azul evitou o aumento do cortisol induzido pelo estresse em $\boldsymbol{O}$. niloticus confinadas por uma hora (manhã e tarde), durante dois dias consecutivos. Uma vez que a perda de peso é indicativa de um estresse crônico nos peixes (MASSOU et al., 2004), a ausência de efeito da cor do ambiente sobre esta variável, neste estudo, pode ser atribuída ao maior tempo de exposição ao estresse.

Observou-se que o estresse de confinamento afetou a CAT e a LDH (Figura 2). A CAT facilita a remoção do peróxido de hidrogênio $\left(\mathrm{H}_{2} \mathrm{O}_{2}\right)$, que é transformado em água e oxigênio molecular, e a redução de sua atividade nos peixes está relacionada à presença de fatores estressantes xenobióticos como pesticidas, que provocam aumento dos radicais superóxidos (TONI et al., 2010; YONAR \& SAKIN,

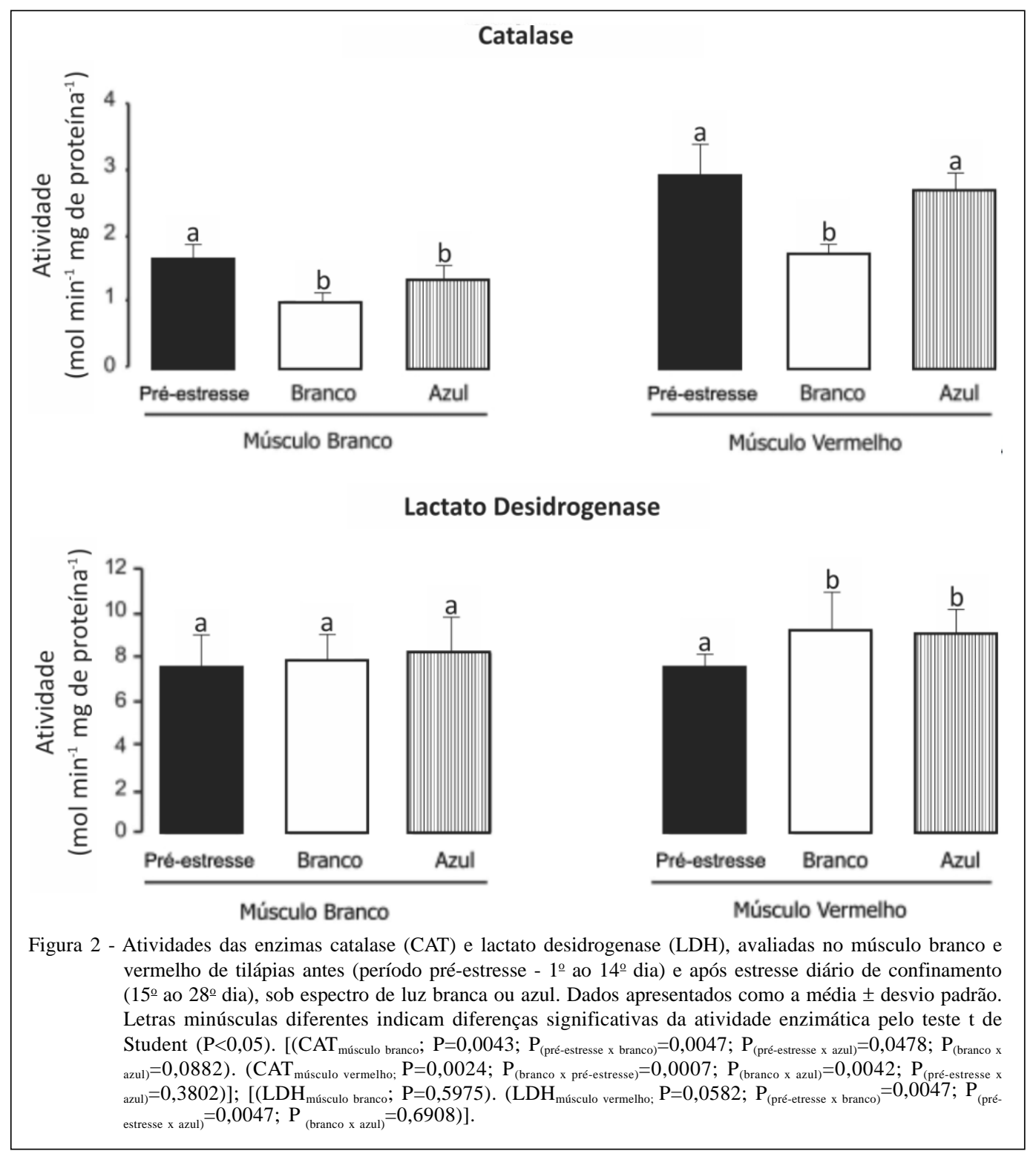

Ciência Rural, v.42, n.5, mai, 2012. 
2011). BRAUN et al. (2006) observaram aumento da atividade desta enzima na espécie Rhamdia quelen, relacionada às baixas concentrações de oxigênio dissolvido na água. No presente trabalho, observouse redução da atividade da CAT da musculatura branca nos peixes submetidos ao estresse, independentemente da coloração do ambiente $\left(\mathrm{P}=0,0043 ; \mathrm{P}_{\text {(pré-estresse } \mathrm{x}}\right.$ branco) $=0,0047 ; \mathrm{P}_{\text {(pré-estresse x azul) }}=0,0478 ; \mathrm{P}_{\text {(branco x azul) }}=0,0882$ ). Na musculatura vermelha, este efeito não foi observado para os peixes mantidos sob luz azul. O estresse provocou redução da atividade da CAT na musculatura vermelha dos peixes mantidos sob a luz branca $\left(\mathrm{P}=0,0024 ; \mathrm{P}_{\text {(branco x pré-estresse) }}=0,0007 ; \mathrm{P}_{\text {(branco x azul) }}=0,0042\right)$, o que não ocorreu entre os peixes no ambiente azul $\left(\mathrm{P}_{\text {(pré-estresse } \mathrm{x} \text { azul) }}=0,3802\right)$, nos quais a atividade desta enzima manteve-se próxima aos níveis basais (controle). Deve-se destacar que a atividade da CAT foi $50 \%$ superior na musculatura vermelha, comparativamente à musculatura branca, nos três tratamentos considerados.

A LDH é uma enzima terminal da glicólise anaeróbia, proporcionando a alguns peixes a habilidade de produzir natações bruscas e repentinas, sendo encontrada em grandes quantidades na musculatura vermelha (BRIGHTMAN et al., 1997). Neste estudo, o estresse não afetou a atividade da LDH na musculatura branca (Figura 2; $\mathrm{P}=0,5975)$, porém aumentou sua atividade na musculatura vermelha $\left(\mathrm{P}=0,0582 ; \mathrm{P}_{\text {(pré-etresse }}\right.$ $x$ branco) $=0,0047 ; P_{\text {(pré-estresse } x \text { azul) }}=0,0047$ ), sem efeito da cor do ambiente $\left(\mathrm{P}_{\text {(branco x azul) }}=0,6908\right)$.

Embora os níveis de cortisol não tenham sido avaliados, pode-se inferir que o confinamento é um agente estressor eficaz, pois reduziu as TCE de todos os peixes. O aumento da atividade da $\mathrm{LDH}$ na musculatura vermelha indica a predominância de processos metabólicos anaeróbios que podem ser relacionados às tentativas de esquiva dos peixes.

\section{CONCLUSÃO}

O confinamento aumenta os processos metabólicos anaeróbios e é adequado para estudos sobre os efeitos do estresse. O espectro de luz azul não evita a redução do crescimento e a demanda energética anaeróbia em situações de estresse, mas preserva a atividade da CAT, contribuindo para o bemestar da tilápia.

\section{COMITÊ DE ÉTICA E BIOSSEGURANÇA}

Este trabalho foi aprovado pelo Comitê de Ética em Experimentação Animal da Faculdade de Zootecnia e Engenharia de Alimentos da Universidade de São Paulo (USP) (Protocolo 160810).

\section{REFERÊNCIAS}

ADAMS, S.M. et al. Evaluating effects of contaminants on fish health at multiple levels of biological organization.Extrapolation from lower to higher levels. Human Ecological Risk Assessment: An International Journal, v.6, n.1, p.15-27, 2000. Disponível em: <http:// w w w. i n f o r m a w o r l d. c o m / s m p p / ftinterface $\sim$ content $=\mathrm{a} 727073024 \sim$ fulltext $=713240930 \sim \mathrm{frm}=$ content $>$. Acesso em: 12 abr. 2011. doi:10.1080/10807030091124428.

AMIYA, N. et al. Effects of background color on GnRH and $\mathrm{MCH}$ levels in the barfin flounder brain. General and Comparative Endocrinology, v.155, p.88-93, 2008. Disponível em: <http://dx.doi.org/10.1016/ j.ygcen.2007.03.007>. Acesso em: 16 abr. 2009. doi: 10.1016/ j.ygcen.2007.03.007.

BARCELLOS, L.J.G. et al. The color of illumination affects the stress response of jundiá (Rhamdia quelen, Quoy \& Gaimard, Heptapteridae. Ciência Rural, v.36, p.1249-1252, 2006. Disponível em: <http://www.scielo.br/pdf/cr/v36n4/ a31v36n4.pdf $>$. Acesso em: 02 jun. 2010. doi: 10.1590/S010384782006000400031 .

BEERS, R.F., SIZER, I.W. A spectrophotometric method for measuring the breakdown of hydrogen peroxide by catalase. Journal of Biological Chemistry, v.195, p.133-140, 1952. Disponível em: <http://www.jbc.org/content/195/1/ 133.full.pdf $>$. Acesso em: 20 maio, 2009.

BERNT, E.; BERGMEYER H.U. Lactate dehydrogenase assay. In: BERGMEYER, H.U. (Ed.). Methods of enzymatic analysis. London: Academic, 1974. p.141-147.

BRIGHTMAN, R.I. et al. Energetics of larval red drum, Sciaenops ocellatus.Part II. Growth and biochemical indicators. Fishery Bulletin, v.95, p.431-444, 1997. Disponível em: <http:// www.marine.usf.edu/bio/physiolab/publications/Energetics-oflarval-red-drum-Part-II.pdf>. Acesso em: 12 abr. 2011.

BRAUN, N. et al. Survival, growth and biochemical parameters of silver catfish, Rhamdia quelen (Quoy \& Gaimard, 1824), juveniles exposed to different dissolved oxygen levels. Aquaculture Research, v.37, p.1524-1531, 2006. Disponível em: <http://onlinelibrary.wiley.com/doi/10.1111/j.13652109.2006.01589.x/pdf $>$. Acesso em: 14 abr. 2011. doi: 10.1111/j.1365-2109.2006.01589.x.

DOOLAN, B.J. et al. Changes in skin colour and cortisol response of Australian snapper Pagrus auratus (Bloch \& Schneider, 1801) to different background colours. Aquaculture Research, v.40, p.542-550, 2009. Disponível em: <http:// dx.doi.org/10.1016/j.ygcen.2007.03.007>. Acesso em: 30 maio, 2010. doi: 10.1016/j.ygcen.2007.03.007.

DOWNING, G.; LITVAK, M.K. The effect of light intensity and spectrum on the incidence of first feeding by larval haddock. Journal of Fish Biology, v.59, p.1566-1578, 2001. Disponível em: <http://onlinelibrary.wiley.com/doi/10.1111/ j.1095-8649.2001.tb00221.x/pdf>. Acesso em: 22 maio, 2010. doi: 10.1111/j.1095-8649.2001.tb00221.x.

FANTA, E. Influence of background color on the behavior of the fish Oreochromis niloticus (Cichlidae). Arquivos de Biologia e Tecnologia, v.38, n.4, p.1237-1251, 1995.

GAINGNON, I.L. et al. Effects de la diminution de la photoperiode sur la smoltification precoce obtenue en 
environnement controle shez le saumon atlantique (Salmo salar). Bulletin français de la pêche et de la pisciculture, v.66, p.307-315, 1993.

HALLIWELL, B.; GUTTERIDGE, J. Free radicals in biology and medicine. Nova York: Oxford University, 2007. V.1, 851p.

HEAD, A.B., MALISON, J.A. Effects of lighting spectrum and disturbance level on the growth and stress responses of yellow perch Perca flavescens. Journal of the World Aquaculture Society, v.31, p.73-80, 2000. Disponível em: <http:// onlinelibrary.wiley.com/doi/10.1111/j.1749. 7345.2000.tb00700.x/pdf $>$. Acesso em: 12 fev. 2010. doi: 10.1 111/j.1749-7345.2000.tb00700.x.

HÖGLUND, E. et al. Behavioural and neuroendocrine effects of environmental background colour and social interaction in Arctic charr (Salvelinus alpinus). Journal of Experimental Biology, v.205, p.2535-2543, 2002. Disponível em: <http://jeb.biologists.org/ cgi/reprint/205/16/2535>. Acesso em: 11 fev. 2010.

JOBLING, M. Fish bioenergetics. London: Chapman-Hall, 1994. 294p.

KARAKATSOULI, N. et al. Combined effects of rearing density and tank colour on the growth and welfare of juvenile white sea bream Diplodus sargus L. in a recirculating water system. Aquaculture Research, v.38, p.1152-1160, 2007. Disponível em: <http://onlinelibrary.wiley.com/doi/10.1111/j.13652109.2007.01780.x/pdf $>$. Acesso em: 16 set. 2010. doi: 10.1111/j.1365-2109.2007.01780.x.

KARAKATSOULI, N. et al. Effects of light spectrum on growth and stress response of rainbow trout Oncorhynchus mykiss reared under recirculating system conditions. Aquacultural Engineering, v.38, p.36-42, 2008. Disponível em: <http:// dx.doi.org/10.1016/j.aquaeng.2007.10.006>. Acesso em: 14 set. 2010. doi: 10.1016/j.aquaeng.2007.10.006.

LINS, P.G. et al. Effect of indole acetic acid administration on the neutrophil functions and oxidative stress from neutrophil, mesenteric lymph node and liver. Life Sciences, v.78, p.564570, 2006. Disponível em: <http://dx.doi.org/10.1016/ j.lfs.2005.04.063>. Acesso em: 13 ago. 2010. doi: 10.1016/ j.lfs.2005.04.063.

MASSOU, A.M. et al. Effects of confinement stress of variable duration on the growth and microincrement deposition in the otoliths of Oreochromis niloticus (Cichlidae). Journal of Fish Biology, v.65, p.1253-1269, 2004. Disponível em: <http:/ / onlinelibrary.wiley.com/doi/ $10.1111 /$ j. 0022 1112.2004.00512.x/pdf $>$. Acesso em: 13 ago. 2010. doi: 10.1111/j.0022-1112.2004.00512.x.

MERIGHE, G.K.F. et al. Efeito da cor do ambiente sobre o estresse social em tilápias do Nilo (Oreochromis niloticus). Revista Brasileira de Zootecnia, v.33, p.828-837, 2004 Disponível em: <http://www.scielo.br/pdf/rbz/v33n4/ 22078.pdf $>$. Acesso em: 20 maio, 2009. doi:10.1590/S151635982004000400002 .

OWEN, M.A.G. et al. Light colour influences the behaviour and stress physiology of captive tench (Tinca tinca). Review of Fish Biology Fisheries, v.20, p. 375-380, 2010. Disponível em: <http:// 10.1007/s11160-009-9150-1. Acesso em: 10 mar. 2010. doi: 10.1007/s11160-009-9150-1.

PAPOUTSOGLOU, S.E. et al. Effects of background color on growth performances and physiological responses of scaled carp
(Cyprinus carpio L.) reared in a closed circulated system. Aquacultural Engineering, v.22, p.309-318, 2000. Disponível em: $\quad<$ http://dx.doi.org/10.1016/S01448609(00)00056-X>. Acesso em: 10 mar. 2010. doi: 10.1016/ S0144-8609(00)00056-X

PAPOUTSOGLOU, S.E. et al. Dietary l-tryptophan and tank colour effects on growth performance of rainbow trout (Oncorhynchus mykiss) juveniles reared in a recirculating water system. Aquacultural Engineering, v.32, p.277-284, 2005. Disponível em: <http://dx.doi.org/10.1016/ j.aquaeng.2004.04.004>. Acesso em: 10 mar. 2010. doi: 10.1016/j.aquaeng.2004.04.004.

RUCHIN, A.B. Influence of colored light on growth rate of juveniles of fish. Fish Physiology and Biochemistry, v.30, p.175-178, $2004 . \quad$ Disponível em: <http:// www.springerlink.com/content/h1m467654507l144/ fulltext.pdf>. Acesso em: 05 ago. 2010. doi:10.1007/s10695005-1263-4.

STATISTICAL ANALYSIS SYSTEM. SAS. User's guide: statistics. Version 8.0. Cary, NC, 1999. 515p.

STOREY, K.B. Oxidative stress: animal adaptations in nature. Brazilian Journal of Medical and Biological Research, v.29, p.1715-1733, 1996. Disponível em: <http://citeseerx .ist.psu.edu/viewdoc/summary?doi=10.1.1.22.9652>. Acesso em: 16 set. 2011. doi: 10.1.1.22.9652.

SUNUMA, T. et al. White background stimulates the food intake of a pleuronectiform fish the barfin flounder, Verasper moseri (Jordan and Gilbert). Aquaculture Research, v.40, p.748-751, 2009. Disponível em: <http:// onlinelibrary.wiley.com/doi/ $10.1111 /$ j. 1365 2109.2009.02179.x/pdf $>$. Acesso em: 19 abr. 2010. doi: 10.1111/j.1365-2109.2009.02179.x

TONI, C. et al. Oxidative stress biomarkers in Cyprinus carpio exposed to commercial herbicide bispyribac-sodium. Journal of Applyed Toxicology, v.30, p.590-595, 2010. Disponível em: <http://onlinelibrary.wiley.com/doi/10.1002/jat.1530/pdf> . Acesso em: 13 abr. 2011. doi: 10.1002/jat.1530.

VAN DER SALM, A.L. et al. Effects of husbandry conditions on the skin colour and stress response of red porgy, Pagrus pagrus. Aquaculture, v.241, p.371-386, 2004. Disponível em: <http:/ /dx.doi.org/10.1016/j.aquaculture.2004.08.038>. Acesso em: 16 jul. 2009. doi: 10.1016/j.aquaculture.2004.08.038.

VOLPATO, G.L., BARRETO, R.E. Environmental blue light prevents stress in the fish Nile tilapia. Brazilian Journal of Medical and Biological Research, v.34, p.1041-1045, 2001. Disponível em: <http://www.scielo.br/pdf/bjmbr/v34n8/ 4190.pdf>. Acesso em: 30 jul. 2010. doi: 10.1590/S0100879X2001000800011.

VOLPATO, G.L. et al. Environmental color affects Nile tilapia reproduction. Brazilian Journal of Medical and Biological Research, v.37, p.479-483, 2004. Disponível em: <http:// www.scielo.br/pdf/bjmbr/v37n4/5083.pdf>. Acesso em: 10 ago. 2009. doi: $10.1590 / \mathrm{S} 0100-879 \times 2004000400004$.

YONAR, M.E.; SAKIN, F. Ameliorative effect of lycopene on antioxidant status in Cyprinus carpio during pyrethroid deltamethrin exposure. Pesticide Biochemistry and Physiology, v.99, p.226-231, 2011. Disponível em: <http:// dx.doi.org/10.1016/j.pestbp.2010.12.008>. Acesso em: 13 abr. 2011. doi: 10.1016/j.pestbp.2010.12.008. 